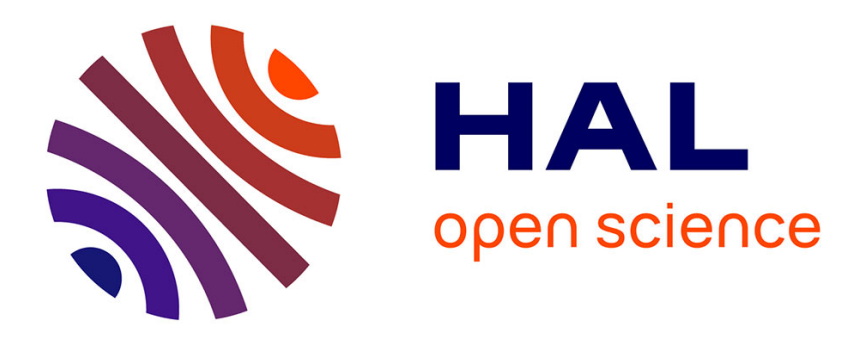

\title{
System of Information Systems as Support for Learning Ecosystem
}

\author{
Majd Saleh, Marie-Hélène Abel
}

\section{To cite this version:}

Majd Saleh, Marie-Hélène Abel. System of Information Systems as Support for Learning Ecosystem. 2nd International Symposium on Emerging Technologies for Education (SETE 2017), Sep 2017, Cape Town, South Africa. hal-01621539

\section{HAL Id: hal-01621539 \\ https://hal.science/hal-01621539}

Submitted on 23 Oct 2017

HAL is a multi-disciplinary open access archive for the deposit and dissemination of scientific research documents, whether they are published or not. The documents may come from teaching and research institutions in France or abroad, or from public or private research centers.
L'archive ouverte pluridisciplinaire HAL, est destinée au dépôt et à la diffusion de documents scientifiques de niveau recherche, publiés ou non, émanant des établissements d'enseignement et de recherche français ou étrangers, des laboratoires publics ou privés. 


\title{
System of Information Systems as Support for Learning Ecosystem
}

\author{
Saleh Majd ${ }^{1}$ and Abel Marie-Hélène ${ }^{1}$ \\ ${ }^{1}$ Sorbonne Universités, Université de Technologie de Compiègne, CNRS \\ HEUDIASYC, UMR 7253, CS 60319 \\ 60203 Compiègne cedex, France \\ \{majd.saleh, marie-helene.abel\}@utc.com
}

\begin{abstract}
Today's learners advance in an environment of fast evolving Information and Communications Technologies (ICT). These learners cannot be supported effectively by the learning strategies of the past. Knowledge is important for the progress of learners, but it is getting harder to obtain with the overwhelming amount resources produced by many Information Systems in the learning ecosystem. In order to manage the knowledge for learners we are following the approach of System of Information Systems (SoIS) as support for learning ecosystem. The SoIS gives the learning ecosystem the opportunity to explore how individual and organizational learning can be enhanced through a combination of different Information Systems in the learning ecosystem. In this context, we look into the orchestration of the SoIS that can provide the ability to index, share, and annotate important resources in the learning ecosystem. In that sense, this paper addresses the approach of System of Information Systems (SoIS) as support of learning ecosystem.
\end{abstract}

Keywords: Learning Ecosystem; System of Information Systems; Collaboration platform.

\section{Introduction}

\subsection{Social Context}

Recently, there has been unprecedented growth in learners' reliance on Information and Communication Technologies (ICT), especially web-based ICTs. Today, nearly most of the viral tasks are carried out by web-based Information Systems. This is made possible as a consequence of the recent growth of communication technologies and Information Systems. As a result, learners depend on many Information Systems to produce, work with, and manage resources. In the process of working at this manner, numerous amounts of digital resources are produced. In overall view, learners deal with huge amount of resources distributed over different Information Systems. In order to reduce this overhead, it would be useful to offer assistance to learners so that they can access the resources in an easy, time saving and organized manner. One of the possible ways in this direction concerns the sharing of information. It is therefore 
interesting to consider the association of different means within the framework of a learning ecosystem in order to produce knowledge from available resources. The goal is to allow learners manage the resources produced by different Information Systems and collaborate with each other over these resources.

\subsection{Scientific Context}

ICT is making many incremental changes to learning ecosystems as information and collaboration systems are introduced to learners on regular basis. This dependency on increasing numbers of Information Systems will require pooling the resources from these different systems to allow for more collaboration [6]. That will create the challenge of managing the huge amount of resources produced in the context of learning ecosystem. In the literature, this challenge is addressed using collaboration tools, recent technologies such as web 2.0 and cloud computing, and knowledge management methodologies. As an attempt to study the relation between learners and the collaboration tools available as web 2.0 technologies, [7] conducted a survey to examine learners' satisfaction and the effectiveness of collaboration systems on the learning experience. It is evident from the results that learners benefit from collaboration systems to access resources anytime and anywhere. The benefit is more when the collaboration system allows for group collaboration and supports digital conversations and discussions.

Regarding the infrastructure, [2] focuses on the advances of cloud computing to the infrastructure of learning ecosystem and the way to store its resources. Opposed to the traditional learning ecosystem, the one empowered by a cloud computing infrastructure allows for more flexible resources management. The key factor is in resources, flexibly, being distributed in many machines and Information Systems connected over a network.

Many Information Systems are being used in the environment of learning ecosystem. According to [5], learners use these systems to generate and share ideas, explore their thinking, and acquire knowledge from other learners. However, due to the failure of indexing methods, learners often fail to reach their desired resources. By using a proper indexing mechanism, learners can plan and select the appropriate resources, based on their own needs and preferences.

We can describe the current situation for learning ecosystems as a group of Information Systems that do not take into account the context of collaboration and the possibility of sharing resources [4]. So, we believe it is necessary to consider:

- The willingness to share resources with a community to achieve a common goal,

- The fact that the shared resources can come from different Information Systems.

In order to meet these needs, we propose to consider a learning ecosystem as a System of Information Systems (SoIS) developed using a collaborative model and introduce new functionalities such as sharing, indexing, annotating and voting for resources. 


\subsection{Plan}

This paper is organized as follows: section 2 presents our approach for managing heterogeneous resources in a learning ecosystem. In section 3, the model of the SoIS is presented. Section 4 contains the prototype of the SoIS (It is called MEMORAeSoIS). Section 5 presents a discussion about the experiment of MEMORAeSoIS prototype with the students at the University of Technology of Compiegne. Finally, this paper is concluded with the prospective ideas and features of the SoIS in section 6.

\section{Our Approach}

In order to find a solution for the problem of overwhelming amount of resources produced within the learning ecosystem, we aim on having a centralized access point where a learner can find, share, and index the resources. Learners can then work and collaborate with these resources. This will save time for the learner and facilitate capitalizing knowledge from different projects and sources. We use the approach of System of Information Systems (SoIS) to implement this central access point as support for the learning ecosystem. The resources accessed by the SoIS can be indexed based on a shared terminology. The learner will have the ability to index resources deemed relevant by the learner who wishes to share them. Learners can then specify why they are relevant through voting and commenting on them. The learner also can share the resources within different sharing spaces containing different members of the learning ecosystem. Other members can easily find the resources indexed and shared with them in their sharing space. All indexed and shared resources presented to the learner by the SoIS may be annotated, commented, and their usefulness can be rated. This enables community driven discussions, for example about the validity of certain resource.

Furthermore, if all the resources can be accessed from centralized platform, added value can be achieved by including a voting functionality.

As mentioned in [8], there are different approaches concerning the coordination of systems in a distributed environment such as SoIS. We choose primarily to focus on the approach of Leader/Follower. In the Leader/Follower approach, a System is designated as the leader of the orchestration of the SoIS while the other systems are designated as followers that can link to the SoIS by following the leader. According to [3], the leader system should provide an added value to the digital ecosystem in which it is operating, by allowing for more communication among the different systems participating in the SoIS. Within the context of our research, the leader system should emphasize on these points:

- Support sharing of resources.

- Support communication of different Information Systems.

- The references for shared resources should be stored and retrieved easily. 
Based on these points we can view the leader system as the organizer of the SoIS. It can be considered to contain a knowledge base that holds all the necessary information of the SoIS (learners' accounts, activities log, references of shared resources, etc.). It should also control the communication line linking all member Information Systems in the SoIS. In addition, it should allow the sharing, indexing, and accessing the resources in the SoIS. Ultimately, the leader system is responsible for providing all the services for the learners in the SoIS to facilitate collaboration and learning process.

Within the environment of the SoIS, overwhelming amount of resources is shared by learners on different Information Systems. The main problem addressed by this paper is how to support the learning ecosystem of learners with the approach of SoIS, and to provide the means to index, share, annotate and vote for resources.

\section{Model}

As mentioned before, the model of SoIS is based on a collaborative model called MEMORAe-core 2 model. The MEMORAe-core 2 collaborative model was developed as part of the MEMORAe project [1]. This project aims to manage heterogeneous resources within organizations and to facilitate organizational learning. Collaboration is considered from the point of view of sharing and exchange heterogeneous resources between collaborators.

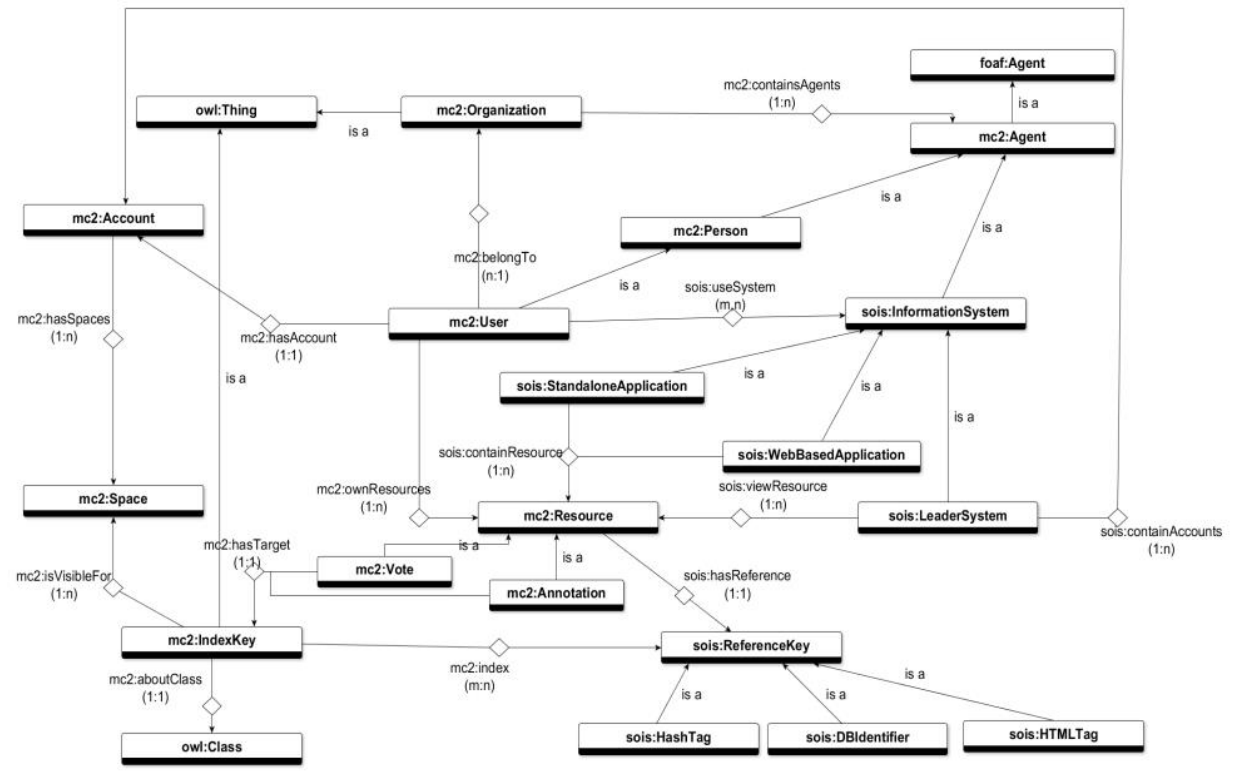

Fig. 1. Model of the SoIS. 
Users are modeled in MEMORAe-core2 by considering an organization as a set of interacting users. Each user can provide support to organizational learning. Each user and group of users is associated with a sharing space where the resources are visible/accessible.

Resources are modeled as "information vectors". Each resource is indexed by one or more indexing keys. A resource can be viewed based on its sharing space and index key.

When examining the model of SoIS it is useful to take into account the representation of resources in MEMORAe-core2 model and build upon it the representation of resources in SoIS. The model is presented in (Fig. 1). The model highlights the position of an agent, either as an Information System or as a user. The model also shows how resources are created by users and contained in the member systems of the SoIS, while the leader system is only showing those resources through a reference key. Each resource has a reference which functions as a link between the resource and the leader system. This key can be an HTML tag, a Database Identifier, or social bookmark as a Hash tag. The leader allows the indexing of references of resources by the index key and sharing it within different sharing spaces.

We also deployed the function of voting so that users evaluate a resource by relevance to its index and sharing space where the resource is visible. As shown in (Fig. 1), vote is a resource in the model of the SoIS. It is related with IndexKey by the relation hasTarget as shown in (Fig. 1).

The SoIS allows the users to interact with various heterogeneous resources coming from different Information Systems in many ways. The user can view the referenced resources, index the important ones, share them within different sharing spaces, annotate, comment and vote on them. An added value can be achieved by tracing the users' activities in the SoIS. The importance of these traces comes from their role in the reasoning process of the recommender system proposed by [10].

In the model of SoIS we can view the leader system as a knowledge base that handles storing, organizing, and sharing of resources from different Information Systems. MEMORAe-core2 model aims, by its design, to facilitate knowledge sharing and capitalization within those different systems. 


\section{MEMORAeSoIS}

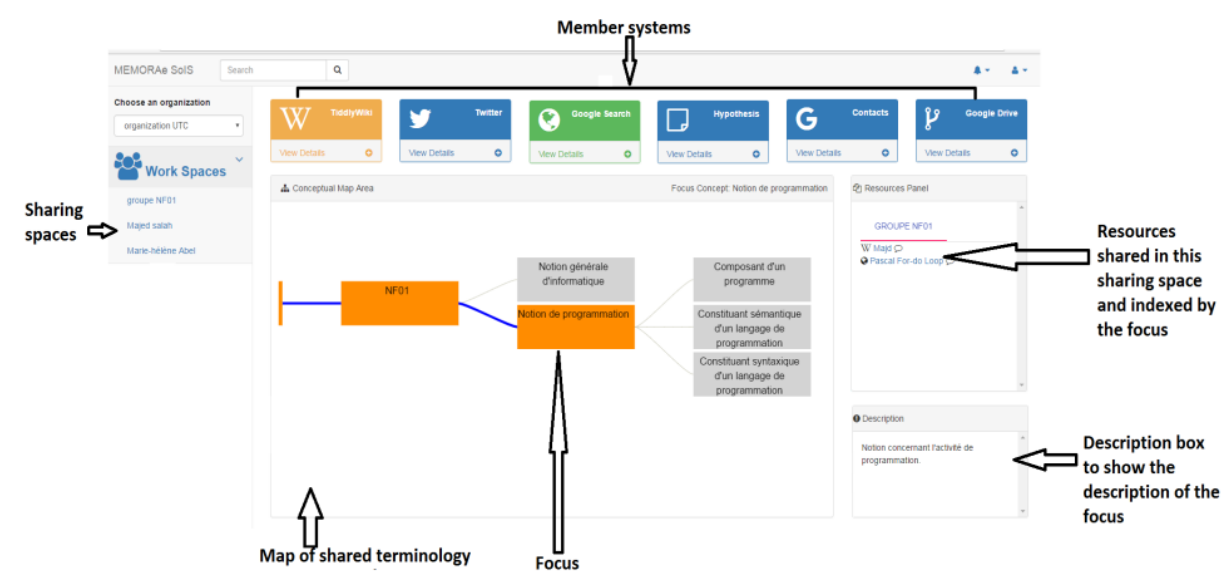

Fig. 2. SoIS Prototype main screen

The member systems of the SoIS are autonomous and work separately of each other. Each of which has its own services and databases. While some systems are openly providing an API for requesting their services, other systems are closed and operate as a black box. Information can be represented in different ways within different systems, thus, the SoIS might face some difficulties accessing resources, unless the services of that system are available through an API. More detailed illustration about the SoIS architecture can be found in [9].The added value of the SoIS is found in the ability to index resources using a map of shared terminology among collaborators and share resources within different sharing spaces. That will give the users of the SoIS the ability to collaborate over resources and rate them. These features are not available when the Information Systems were working separately. In the premise of the SoIS the learners can share resources from different external systems and vote on them with simplicity and ease.

Other than the leader system the prototype links various Information Systems that are different in their purpose and goals, hence they produce different types of resources. These systems are: TiddlyWiki (wiki pages content management), Twitter (search for tweets by hashtag), Google Search (search the web using google search engine), Hypothesis (web page annotation tool), Google Contacts (your google contact), Google Drive (file upload and storage). 


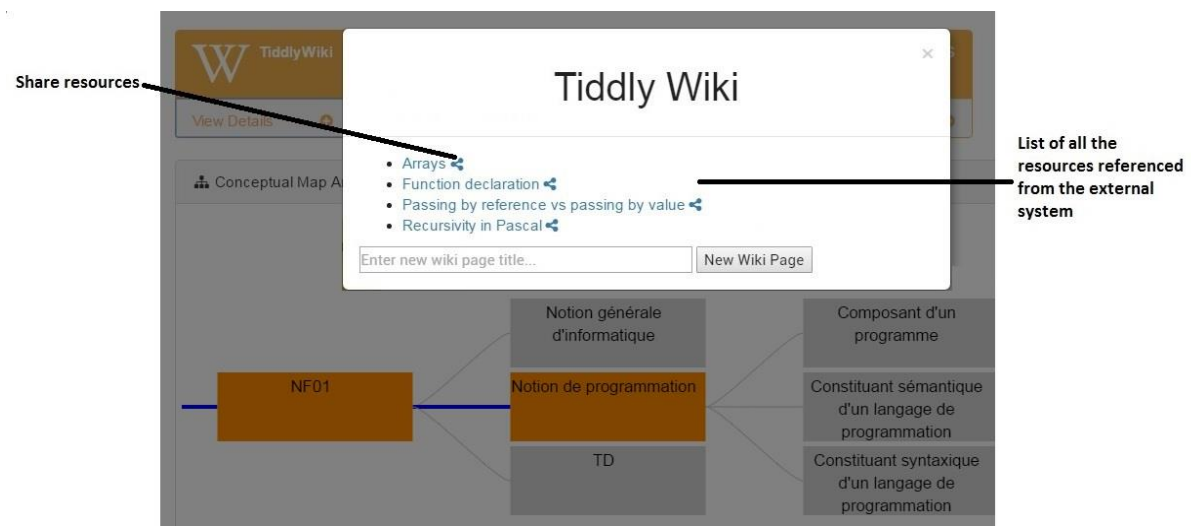

Fig. 3. Panel to access resources available in the TiddlyWiki, or create new ones.

The purpose of the prototype is to provide hands-on experience for our solution to the problem of managing resources in learning ecosystem, sharing, voting for, and annotating these resources with the help of a map of shared terminology. After login, the learner can choose an organization that he/she belongs to. The learner might have one or more organizations to choose from. Based on the chosen organization, the SoIS will view a map specific to that organization and can be used to index the heterogeneous resources. The learner will also have access to the sharing spaces. For each learner there is at least two sharing spaces; one global for the whole organization and the other is the personal sharing space. Resources of the member systems are referenced in their respected tabs. The learner can view all the referenced resources for each system and index them by an index key (the index key represents a node in the map of shared terminology), and share the indexed resources in a sharing space. Moreover, on each box that is used to access a member system there is a button with a "plus" sign. This button will allow the user to navigate through all the resources available in the dedicated Information System and select from the list of resources which are going to be indexed by the map and shared in certain sharing spaces. The panel to navigate through resources is shown in (Fig. 3).

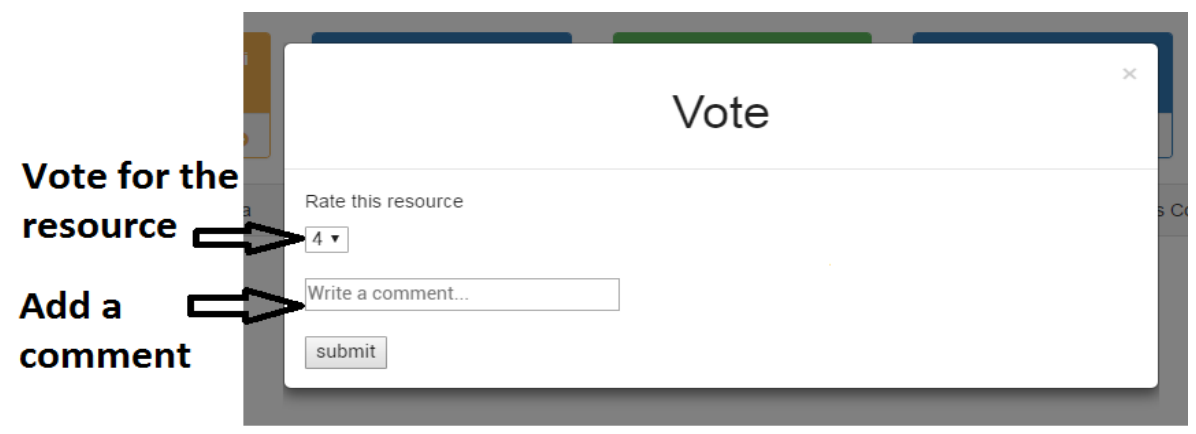

Fig. 4. Vote and comment panel. 
After indexing the important resources, users are able to annotate and vote for those resources. The annotation and votes are themselves considered as resources. They, as well, can be indexed and shared in their own right.

In the platform, you can vote and comment on a resource by clicking the icon next to the indexed resource. Then you can choose a score (1 to 5) from the dropdown list, and click the vote button. You can also add a comment about this resource to justify your vote as seen in (Fig. 4).

\section{Discussion}

For the prototype to be tested we currently collect feedback data from students at the University of Technology of Compiegne (UTC) to evaluate the usefulness of MEMORAeSoIS. The students are enrolled in a computer science course focused on the subjects of algorithms and programming. We will use their feedback on MEMORAeSoIS based on two aspects; a survey form, and hands-on sessions with the platform. For the setup of this test, each student is provided with an account. This account has access to a map of concepts representing the course in which students are enrolled. Students then can index, share, annotate, and vote on resources related to this course. Also, each student has three sharing spaces in the platform: a personal sharing space, a sharing space for the group in which the student belongs, and a last sharing space with the instructor of the course.

Students at UTC usually have access to an online course management and collaboration platform called Moodle. Moodle benefits the students with a learning environment that supports assignment submission, discussion forum, files download, grading, instant messages, online quizzes, and wiki. The aforementioned features help a student to post queries, search for information, read daily posts and comments, and lastly takeâ€"up an online quiz. In overall view, Moodle provides an encompassing solution for collaboration and course management in a learning ecosystem. What is missing here is a mechanism for sharing and indexing resources in a way that makes it easy for students to find and work with. MEMORAeSoIS provides this mechanism on top of its ability to connect the learner with different Information Systems that can help him/her with the learning process. MEMORAeSoIS introduces the idea of a map of shared terminology that is used to index the resources in the learning ecosystem. Furthermore, resources from Moodle can indexed and shared within MEMORAeSoIS as weblinks as seen in (Fig. 5).

The students themselves participate in the construction of the map in MEMORAeSoIS. This is also a positive point in favor of MEMORAeSoIS, as the map comes out based on students' needs and requirements. the map represents the notions to be covered in the course. It is a representation of the course content. The map was defined by the instructors. During the course, the students asked to expand the map with a branch intended for tutorials (TDs) in order to be able to index the resources related to the contents of these TDs. They can thus access the resources by the TD or by the topic treated in the TD; for example: The third TD covers the topic of conditional 
statements in pascal. When the students want to index resources related to this topic they can index them by the node "TD3" and the node "conditional statements".

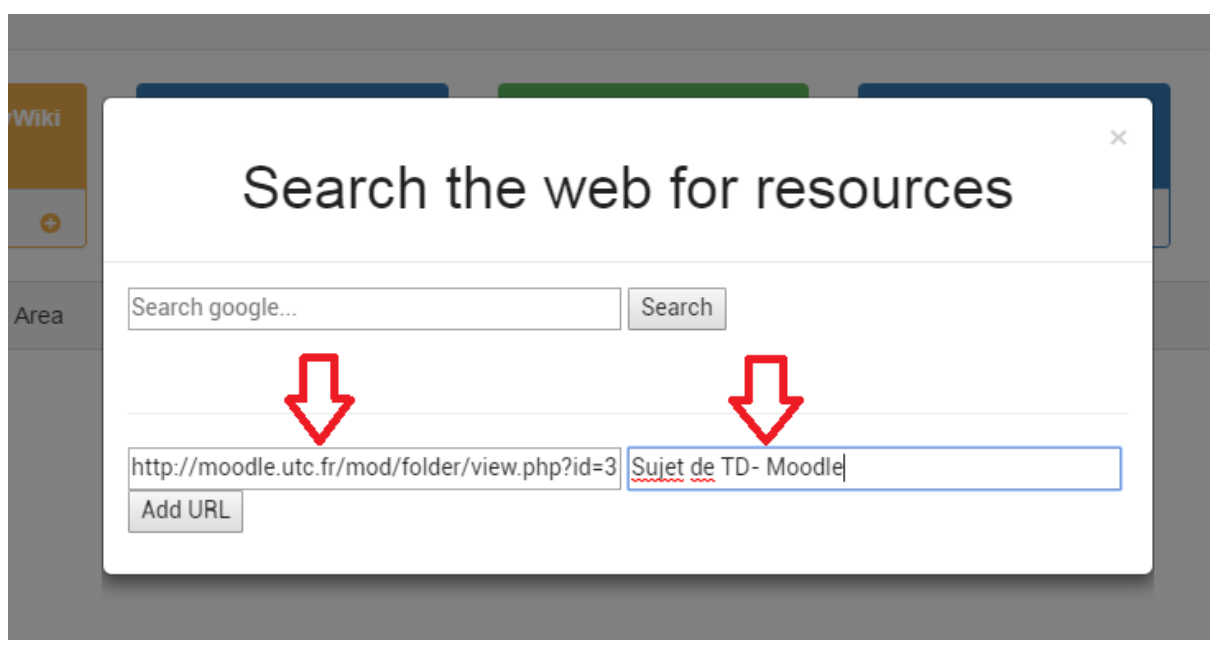

Fig. 5. Add resources from Moodle in MEMORAeSoIS.

\section{Conclusion and Future Work}

The goal of this paper was modeling and developing a System of Information Systems as support for learning ecosystem. The resources are the result of different learners working on different projects, using several Information Systems in the digital environment of a learning ecosystem. The aim was focused towards facilitating resources management in a System of Information Systems (SoIS), and to model and develop MEORAeSoIS. To achieve this goal this paper undertakes an effort to present the social and scientific context of this research and define the state of the art, then move to present our approach that helped in realizing the model of the SoIS composed of various Information Systems with the inspiration of MEMORAe-core 2 collaborative model. This paper found potentials in adapting the MEMORAe-core2 to manage the resources produced by different systems in the SoIS. It was also clear that combining resources from various Information Systems and manage them within the leader system will result in an added value to learners not present when those systems were operating separately. The added value that we aim to achieve in MEMORAeSoIS prototype is the ability to index, share, annotate, vote, and comment on resources. Furthermore, the SoIS can upgrade this value by tracking the activities of learners and providing analysis of these activities to determine learners' competence levels at certain subjects. The prototype is being tested by the students at the University of Technology of Compiegne (UTC) to evaluate the usefulness of MEMORAeSoIS.

The next step is to expand our work to implement the learners' trace and introduce new Information Systems to MEMORAeSoIS based on the prototype presented in this paper and learners' needs. MEMORAeSoIS should keep simple interface, with all the 
resources as far from the user as a single click, to keep the users experience useful and friendly.

\section{$7 \quad$ Acknowledgement}

This project is done under ECOPACK project and funded by ANR-13-ASTR-0026 program.

\section{References}

1.Abel, M.H.: Knowledge map-based web platform to facilitate organizational learning return of experiences. Computers in Human Behavior 51, 960-966 (2015)

2.Dong, B., Zheng, Q., Yang, J., Li, H., Qiao, M.: An e-learning ecosystem based on cloud computing infrastructure. In: Advanced Learning Technologies, Ninth IEEE International Conference on. pp. 125-127. IEEE (2009)

3.Dong, H., Hussain, F.K.: Digital ecosystem ontology. In: Industrial Electronics, IEEE International Symposium on. pp. 2944-2947. IEEE (2007)

4.García-Peñalvo, F.J., Hernández-García, Á., Conde, M.Á., Fidalgo-Blanco, Á., SeinEchaluce, M.L., Alier, M., Llorens-Largo, F., Iglesias-Pradas, S.: Learning services-based technological ecosystems. In: Proceedings of the 3rd International Conference on Technological Ecosystems for Enhancing Multiculturality. pp. 467-472. ACM (2015)

5.Lau, A., Tsui, E.: Knowledge management perspective on e-learning effectiveness. Knowledge-Based Systems 22(4), 324-325 (2009)

6.Laurillard, D.: E-learning in higher education. Changing higher education: The development of learning and teaching pp. 71-84 (2006)

7.Liaw, S.S.: Investigating students perceived satisfaction, behavioral intention, and effectiveness of e-learning: A case study of the blackboard system. Computers \& Education 51(2), 864-873 (2008)

8.Lozano, R., Spong, M.W., Guerrero, J.A., Chopra, N.: Controllability and observability of leader-based multi-agent systems. In: Decision and Control, 47th IEEE Conference on. pp. 3713-3718. IEEE (2008)

9.Majd, S., Marie-Hélène, A., Véronique, M., Claude, M., David, V.: Integration of brainstorming platform in a system of information systems. In: Proceedings of the 8th International Conference on Management of Digital EcoSystems. pp. 166-173. ACM (2016)

10. Wang, N., Abel, M.H., Barthes, J.P., Negre, E.: Mining user competency from semantic trace. In: Computer Supported Cooperative Work in Design (CSCWD), IEEE 19th International Conference on. pp. 48-53. IEEE (2015) 